\title{
Quantifying and reducing spurious alignments for the analysis of ultra-short ancient DNA sequences
}

\author{
Cesare de Filippo* (D), Matthias Meyer and Kay Prüfer ${ }^{*}$
}

\begin{abstract}
Background: The study of ancient DNA is hampered by degradation, resulting in short DNA fragments. Advances in laboratory methods have made it possible to retrieve short DNA fragments, thereby improving access to DNA preserved in highly degraded, ancient material. However, such material contains large amounts of microbial contamination in addition to DNA fragments from the ancient organism. The resulting mixture of sequences constitutes a challenge for computational analysis, since microbial sequences are hard to distinguish from the ancient sequences of interest, especially when they are short.

Results: Here, we develop a method to quantify spurious alignments based on the presence or absence of rare variants. We find that spurious alignments are enriched for mismatches and insertion/deletion differences and lack substitution patterns typical of ancient DNA. The impact of spurious alignments can be reduced by filtering on these features and by imposing a sample-specific minimum length cutoff. We apply this approach to sequences from four 430,000-year-old Sima de los Huesos hominin remains, which contain particularly short DNA fragments, and increase the amount of usable sequence data by $17-150 \%$. This allows us to place a third specimen from the site on the Neandertal lineage.
\end{abstract}

Conclusions: Our method maximizes the sequence data amenable to genetic analysis from highly degraded ancient material and avoids pitfalls that are associated with the analysis of ultra-short DNA sequences.

Keywords: Ancient DNA, Spurious alignments, Sima de los Huesos

\section{Background}

After its death, the DNA of an organism inevitably degrades into short DNA fragments [1,2]. Laboratory methods have been developed that specifically aim at retrieving these fragments from ancient biological material [3-5] and transforming them efficiently into library molecules for high-throughput sequencing [6]. These developments have enabled researchers to study DNA sequences from increasingly older samples. One notable example are four remains from Sima de los Huesos in Spain that constitute, with an age of over 400,000 years, the by far oldest hominin material to date that yielded ancient DNA sequences $[7,8]$. Owing to their great age, the vast majority of hominin DNA fragments that can

\footnotetext{
*Correspondence: cesare_filippo@eva.mpg.de; pruefer@eva.mpg.de Max Planck Institute for Evolutionary Anthropology, 04103 Leipzig, Germany
}

be extracted from the Sima de los Huesos remains are shorter than 45 bp [7].

In addition to the extreme state of DNA fragmentation, the analysis of sequences from highly degraded material is hampered by the large number of extraneous DNA fragments originating from microorganisms that decomposed the remains of the source organism after its death [9-12]. In the case of Sima de los Huesos [8] and many other ancient skeletal remains, microbial DNA constitutes more than $99 \%$ of the DNA that can be recovered and sequenced. Contaminant sequences are typically differentiated from those that stem from the source organism by aligning all sequences to a related reference genome and retaining only those that produce alignments with not more than a pre-defined number of differences [13, 14]. However, unrelated sequences can align by chance and the probability of such spurious alignments increases with decreasing sequence length [15]. 
This issue is expected to affect particularly the analysis of sequences from highly fragmented material.

To minimize the effect of spuriously aligning sequences on downstream analyses, previous studies employed sequence length cutoffs that have been gauged by a variety of methods. Green et al. [16] used specific alignment software to analyze the distribution of alignment scores at various sequence lengths. This distribution was found to be distinctly bimodal at longer lengths, as expected from a mixture of related and unrelated sequence alignments, while bimodality was not observed at shorter lengths. Setting a length cutoff that preserves the bimodal distribution can thus be used to limit the fraction of spurious alignments. Cutoffs have also been determined by testing at which lengths mammoth sequences yielded equally good alignments to other mammalian taxa $[10,13]$, horse sequences aligned equally well to the chicken genome [17], mammoth and ancient bovine sequences aligned to a database of concatenated bacterial genomes [18], or fragmented bacterial genomes aligned to the human reference [19]. While these methods have been sufficient to determine approximate cutoffs, they do not provide an estimate of the fraction of spurious alignments. We also note that microbial genomes in public databases may present a poor proxy for the microbial sequence diversity found in real sequence data from ancient remains. The validity of these approaches is therefore hard to judge.

More recently, Meyer et al. [8] used a different approach to determine sequence length cutoffs for the analysis of nuclear DNA sequences from the Sima de los Huesos samples. Using sequence variants that are unique to the human reference genome, as determined by comparison to known variation from human resequencing studies and the genomes of non-human primates, they counted the fraction of sequences that match the reference-specific variant. These variants are rare and are expected to be largely absent in other hominin genomes. In contrast, spuriously aligned sequences will match the reference genome by chance, independent of how frequent the reference genomes' variants are in the human population. Since no matches to the reference-specific variant was observed for sequences of at least 35-bp length, this cutoff was deemed sufficient to exclude spurious alignments. However, due to the limited number of unique reference variants (i.e., 11,299) and the small amount of data obtained from the Sima de los Huesos remains (less than 0.001 -fold genomic coverage per sample), only between 4 and 69 sequences formed the basis for this assessment, preventing any fine-scale estimates of the fraction of spurious alignments.

Here, we test and extend this approach to allow for the confident estimation of the fraction of spurious alignments across different sequence lengths. We use these estimates to devise sequence length cutoffs that maximize the number of useful sequences and increase the power of phylogenetic analysis. Applying our approach to the Sima de los Huesos samples, we determine that cutoffs shorter than $35 \mathrm{bp}$ are suitable for some of these samples, as long as appropriate filters are put in place. The increase in usable sequences allows us to confidently place one of the Sima de los Huesos samples on the Neandertal lineage that previously yielded inconclusive results.

\section{Results}

\section{Estimating the fraction of spurious alignments}

To allow for fine-scale estimates of the fraction of spurious alignments in small datasets, we changed $\sim 18$ million interspersed bases in the human reference genome (see the "Methods" section). These artificial mutations were introduced at positions where the human reference, all human genomes sequenced as part of the 1000 Genomes project, two high-coverage archaic human genomes, and the chimpanzee genome show the same base. They are thus unlikely to occur in present-day or ancient hominin genomes (probability $<0.1 \%$ ). Spurious alignments, on the other hand, are likely to match the mutated state (Fig. 1a). The alignment parameters used here and in other studies [14,20] limit the fraction of allowed mismatches per alignment to approximately $10 \%$ (see the "Methods" section), resulting for spuriously aligned sequences in a predicted $\sim 90 \%$ match probability for the mutated state and a $\sim 3.3 \%$ probability for matching either of the remaining three states (Fig. 1a).

To test whether these predictions hold, we generated sequences from DNA isolated from the blood sample of a healthy human individual that was fragmented heavily to mimic the size distribution of ancient DNA. We further compiled a dataset consisting of 3860 bacterial genomes that were cut in silico into 3.03 billion unique sequences uniformly distributed between 20- and 40-bp length (see the "Methods" section). We then mapped both sets of sequences to the mutated reference and counted the fraction of sequences that match the reference state at mutated positions (presumed hominin alignment, henceforth "true alignment") or any other variant (presumed "spurious alignment"). Of the aligned human sequences, $99.8 \%$ were correctly classified as hominin. Out of 782 million bacterial sequences that could be aligned to the mutated reference, $97.6 \%$ were correctly classified as spurious (Additional file 1: Table S1). If all alignments of bacterial sequences contained the maximal number of allowed mismatches, $3.9 \%$ of the sequences would be expected to carry the reference state by chance, whereas we observe a lower percentage of $2.4 \%$ (see Additional file 1: Tables S2 and S3 for a similar analysis with cut sequences from a protist and a fungus genome). Since the percentage of misclassified sequences 
a

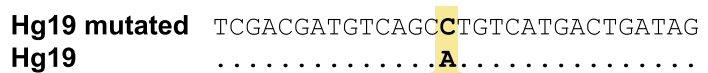

Hg19

possible

aligned sequences

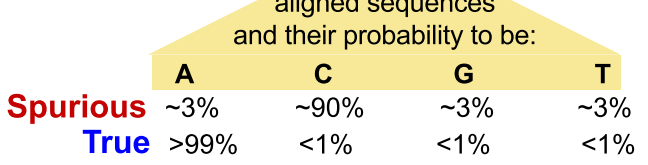

b

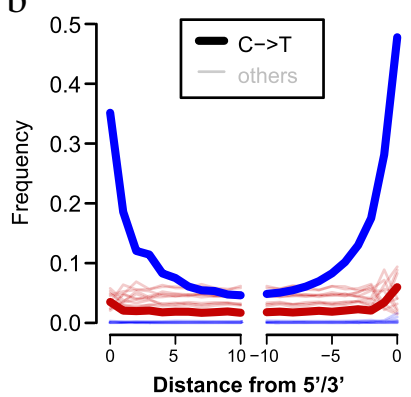

c

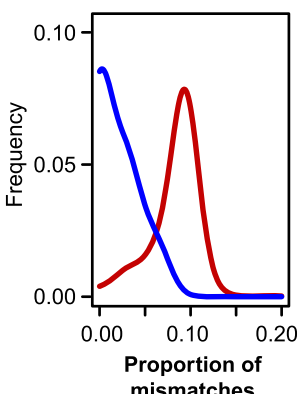

d

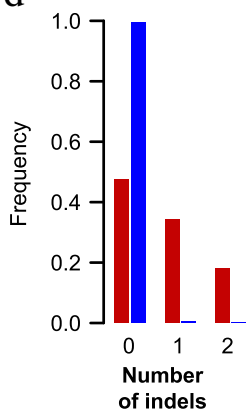

Fig. 1 Identification and characterization of spurious and true sequence alignments. a Schematic illustration of how spurious and true sequence alignments are inferred. The human reference (hg19) is mutated to introduce changes at positions that are not known to vary among presentday humans and other hominins. True hominin sequences (blue) and spuriously aligned microbial sequences (red) are expected to show the reference, the mutated or one of the two other states with the probabilities indicated. b Frequency of all nucleotide substitutions at each position in Mezmaiskaya 1 sequence alignments. c Distributions of the proportion of mismatches in Mezmaiskaya 1 alignments. One mismatch was subtracted from all true alignments and those spurious alignments that did not carry the mutated allele. This was done to compensate for the fact that these alignments have to carry a mismatch to the mutated reference genome in order to be identified as such. $\mathbf{d}$ Distributions of the number of indels in Mezmaiskaya 1 alignments. See Additional file 1: Figure S1 for the distribution of mismatches and indels with the modern human and the bacterial datasets that were used as negative and positive controls, respectively

biases the estimated fraction of spurious alignments slightly downward, we corrected our estimates in all subsequent analyses using conservatively the expected proportion (see the "Methods" section).

\section{Characteristics of spurious and true alignments}

We next investigated whether spurious and true alignments differ in specific characteristics. For this purpose, we aligned sequences from the Mezmaiskaya 1 Neandertal [20, 21], a published dataset containing a considerable fraction of ultra-short ( $<35 \mathrm{bp}$ ) sequences (Additional file 1: Figure S2) and approximately 9\% Neandertal DNA, to the mutated reference. After filtering for mappability (see the "Methods" section) and classifying the alignments as described above, we obtained 5.07 million true alignments and 0.92 million spurious alignments. The procedure uses strand orientation to avoid misclassifying sequences due to ancient DNA damage (see the "Methods" section).

We first note that true Mezmaiskaya 1 sequence alignments show elevated frequencies of C-to-T substitutions, which occur predominantly at their beginning and ends (Fig. 1b). This pattern is expected for authentic ancient DNA sequences and results from deamination of cytosine to uracil in single-stranded DNA overhangs [22].
In contrast, this pattern is not observed for spurious alignments, where C-to-T substitutions are similar in frequency to other types of substitutions. Second, we find that true alignments carry significantly fewer mismatches on average than spurious alignments $(0.018$ vs 0.108 per bp; Wilcoxon rank sum test $p$ value $<2.2 \mathrm{e}-16$; see Fig. $1 \mathrm{c})$. The fraction of mismatches in the true alignments is still substantially larger than the genomic divergence between modern humans and Neandertals of $<0.002$ differences per base pair [21]. However, C-to-T substitutions account for most of this difference (Fig. 1b). Third, true alignments contain fewer insertions/deletions (indels) than spurious alignments $(0.5 \%$ vs. $52.4 \%$ of the alignments, Wilcoxon rank sum test, $p$ value $<2.2 \mathrm{e}-16$ ) (Fig. 1d). Indels accumulate at a roughly 10 times lower rate than single nucleotide mutations in humans [23] and are therefore expected to be rare in true alignments.

We repeated these analyses using the bacterial and modern human control datasets (Additional file 1: Figure S1 and Table S4). Similar to the results from spurious Mezmaiskaya 1 alignments (Fig. 1c, d), bacterial alignments are enriched for mismatches (0.092 per bp on average) and indels (76.2\% of the alignments), whereas mismatches and indels are rare among modern human control alignments ( 0.004 per bp and $0.05 \%$, respectively). 


\section{Minimizing the proportion of spurious alignments}

We next binned all Mezmaiskaya 1 sequences by length and calculated the fraction of spurious alignments for each bin. As expected, the fraction of spurious alignments increases with decreasing sequence length (Fig. 2). Spurious alignments are rare $(<0.3 \%)$ in sequences of at least $35 \mathrm{bp}$ length, suggesting that a sequence length cutoff of $35 \mathrm{bp}$, which was used in several ancient DNA studies (Additional file 1: Table S5), is effective in removing the vast majority of spurious alignments for the Mezmaiskaya 1 dataset analyzed here. In fact, even sequences of length 33 bp show a proportion of spurious alignments of less than $1 \%$, indicating that shorter sequences could be included in downstream analyses (Fig. 2).

The previous analysis has shown that spurious alignments lack the elevation of terminal C-to-T substitution frequencies that are typical for ancient DNA and that they contain more indels than true alignments (Fig. 1). Filtering based on these features may thus help to further reduce the fraction of spurious alignments. In agreement with this assumption, we find that restricting the analysis to alignments exhibiting a C-to-T substitution at either terminus yields less than $1 \%$ spurious alignments for length bins as short as $30 \mathrm{bp}$. It should be noted that this deamination filter is often used to deplete sequence data of human contamination. However, it also removes a large fraction of potentially genuine ancient sequences that were not affected by deamination $(\sim 85 \%$ of aligned sequences $\geq 35$ bp in Mezmaiskaya 1). A less pronounced effect is observed when removing alignments with indels $(\sim 1 \%$ of aligned sequences $\geq 35$ bp in Mezmaiskaya 1), which yields less than $1 \%$ spurious alignments in size bins of 32 bp or longer. Combining both filters reduces this number to $29 \mathrm{bp}$. The reduction of spurious alignments achieved with both filters is also reflected by a decrease in sequence differences to the reference genome (Fig. 2).

We repeated this analysis using our modern human control sample, which should, by design, not produce any spurious alignments. We find that even the shortest length bin yields an estimate for the proportion of spurious alignments of less than 2\% (Fig. 2), suggesting that sequencing or mapping errors have little impact on our measure.

\section{A re-analysis of sequences from Sima de los Huesos}

The extremely short DNA sequences that have been retrieved from the Sima de los Huesos remains are an ideal dataset to explore to which extent the choice of sequence filters changes the amount of useful sequence

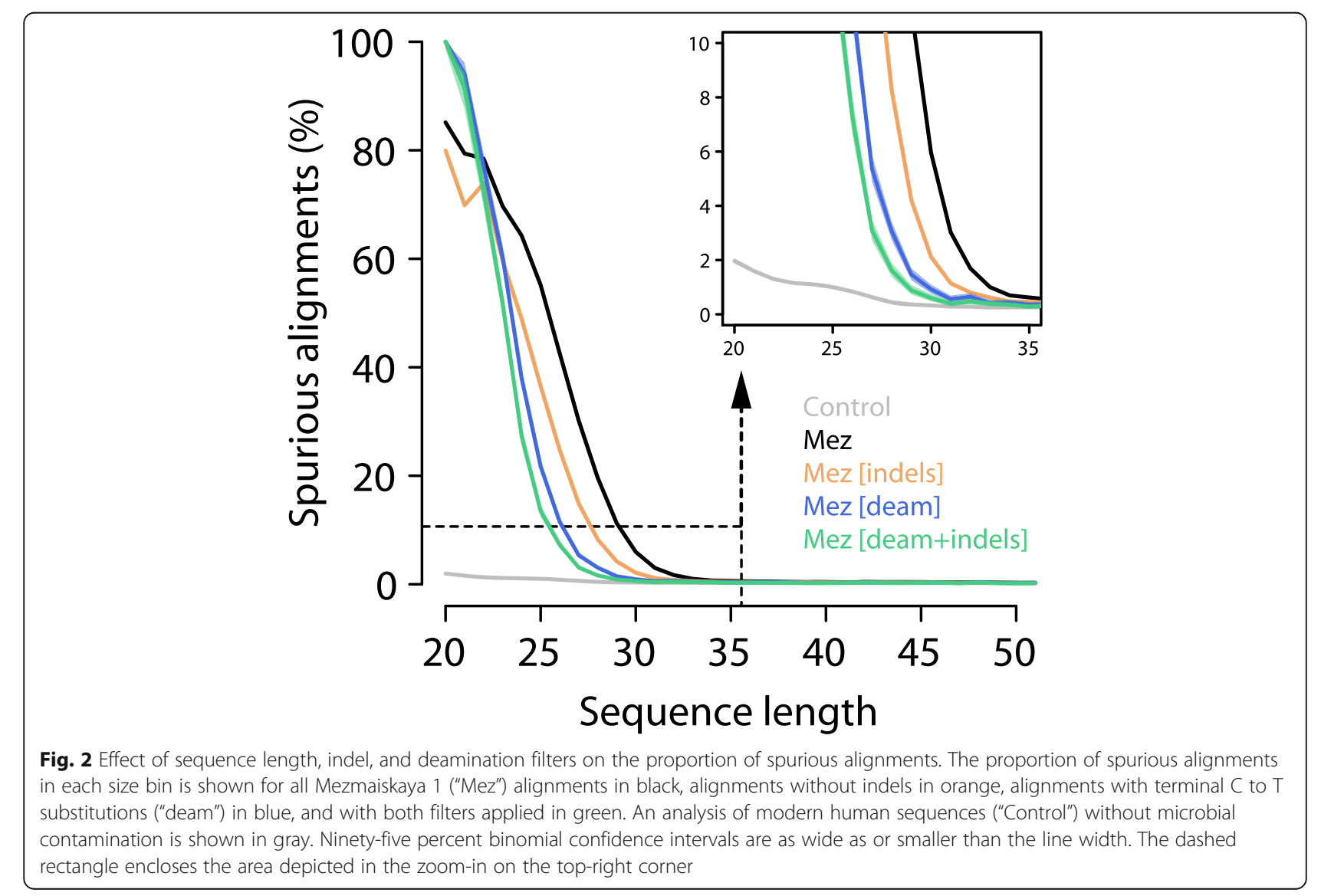


data that can be obtained from very poorly preserved material and the inferences that can be drawn from these data. Appreciable amounts of nuclear DNA sequences are available from four hominin remains from the site [8]; the fraction of hominin DNA varies between 0.02 and $0.18 \%$ in these samples when considering sequences of at least 35-bp length. However, the vast majority (>97\%) of the human aligned sequences of at least 20-bp length are shorter than this 35-bp cutoff (Additional file 1: Figure S2).

To determine whether at least some of these ultra-short sequences are amenable to analysis, we realigned the data of all four samples to the mutated reference genome and removed alignments that contained indels and those showing no evidence of deamination (see also Additional file 1: Figures S3 and S4). The deamination filter is strictly required when working with these data, as a substantial fraction of the hominin sequences is derived from modern human contamination $[7,8]$. We then calculated sequence length cutoffs that limit the fraction of spurious alignments to $<1 \%$ or $<10 \%$, henceforth denoted by $L_{1 \%}$ and $L_{10 \%}$, respectively.

The four samples yield $L_{10 \%}$ cutoffs that range from 27 to $34 \mathrm{bp}$ and decrease with increasing proportions of endogenous DNA (Fig. 3, Table 1). Applying these cutoffs instead of the previously used cutoff of $35 \mathrm{bp}$ would increase the usable data by $17-150 \%$. The more conservative $L_{1 \%}$ cutoffs would result in $0-40 \%$ more data for three of the four samples. Interestingly, the fourth sample, FemurXIII, yields a $L_{1 \%}$ cutoff of $46 \mathrm{bp}$, suggesting that the often applied cutoff of $35 \mathrm{bp}$ is not always sufficient to limit spurious alignments to low levels. In comparison, sequences from the Mezmaiskaya 1 Neandertal yield an $L_{1 \%}$ of $22 \mathrm{bp}$ and do not reach a limit for $L_{10 \%}$ (less than $10 \%$ of all sequences of at least 20-bp length aligned spuriously). Considering sequences of at least $20 \mathrm{bp}$ for analysis would result in $37 \%$ more data compared to a 35-bp length cutoff.

Since present-day human contamination constitutes a challenge for the analysis of archaic human sequences, we also tested whether contamination rates differ when including shorter sequences. We found no significant differences in the estimated contamination compared to the previously used length cutoff of $35 \mathrm{bp}$, although this result may be caused by a lack of power for the Sima de los Huesos samples (Additional file 1: Table S6). We note that contamination estimates tend to be higher using $L_{10 \%}$ cutoffs likely due to a reference bias, causing spurious alignments to match the human reference allele more likely than the archaic allele.

We also note that non-human eukaryotic contaminants would not be expected to be enriched among shorter sequences since contaminant sequences tend to be longer and the reference bias acts against their alignment [24].

\section{Improving phylogenetic inferences from limited data}

The initial analysis of nuclear DNA sequences from the Sima de los Huesos specimens revealed that two of the

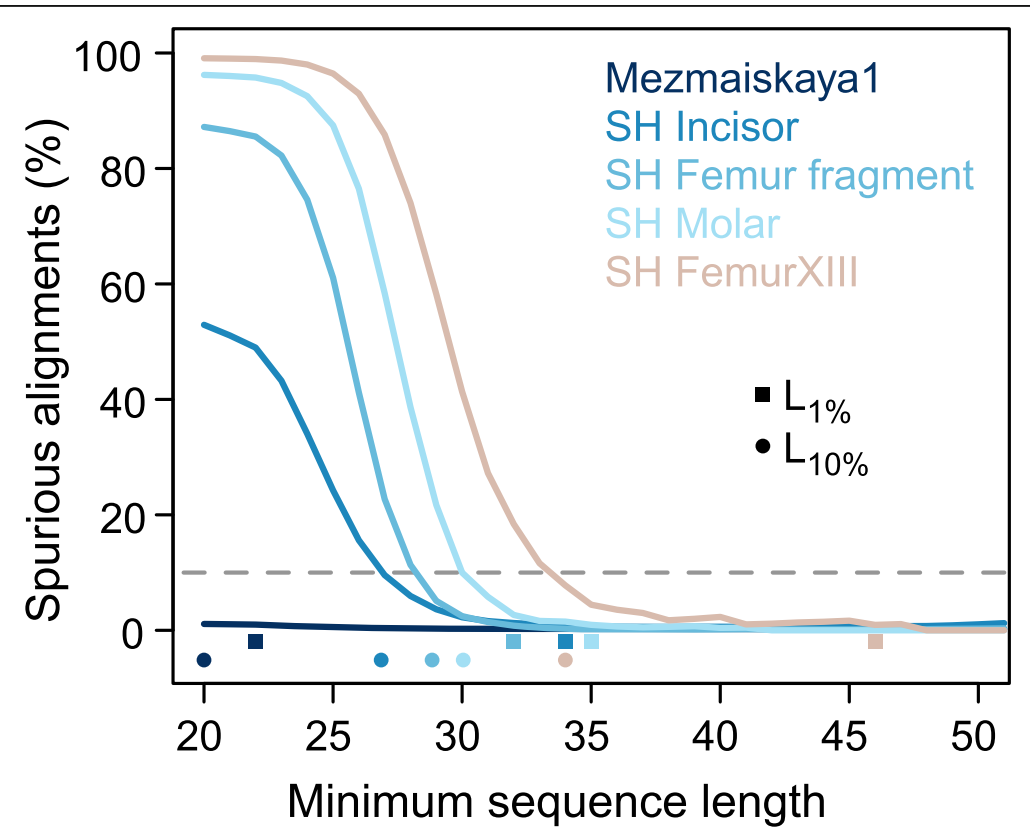

Fig. 3 Cumulative proportion of spurious alignments. Squares and dots on the $x$-axis show the length-cutoffs that guarantee a spurious alignment rate lower than $1 \%\left(L_{1 \%}\right)$ and lower than $10 \%\left(L_{10 \%}\right)$, respectively (see also Table 1$)$. The dashed horizontal gray line indicates $10 \%$ spurious alignments. Only sequences with C-to-T changes in the terminal $5^{\prime}$ and $3^{\prime}$ positions and without indels are considered (i.e., the filters "deam+indels" used in Fig. 2) 
Table 1 Mezmaiskaya and Sima de los Huesos (SH) sequence length cutoffs allowing for less than 1\% or 10\% spurious alignments

\begin{tabular}{|c|c|c|c|c|c|c|c|c|}
\hline \multirow[t]{2}{*}{ Samples } & \multirow{2}{*}{$\begin{array}{l}\text { Hominin } \\
\text { DNA (\%) }\end{array}$} & \multicolumn{2}{|c|}{ Length cutoff $(\mathrm{bp})^{\mathrm{b}}$} & \multicolumn{3}{|c|}{ Total number of hominin bases recovered (Mbp) } & \multicolumn{2}{|c|}{ Fold change } \\
\hline & & $\overline{L_{1 \%}}$ & $L_{10 \%}$ & $35 b p$ & $L_{1 \%}$ & $L_{10 \%}$ & $L_{1 \%} / 35 \mathrm{bp}$ & $L_{10 \% / 35 ~ b p}$ \\
\hline Mezmaiskaya 1 & 8.84 & 22 & 20 & 88.53 & 121.17 & 121.73 & 1.37 & 1.37 \\
\hline SH Femur frag. & 0.11 & 32 & 29 & 0.81 & 1.14 & 1.52 & 1.41 & 1.88 \\
\hline SH Incisor & 0.18 & 34 & 27 & 1.49 & 1.70 & 3.71 & 1.14 & 2.49 \\
\hline SH Molar & 0.03 & 35 & 30 & 0.13 & 0.13 & 0.25 & 1.00 & 1.98 \\
\hline SH FemurXIII & 0.02 & 46 & 34 & 0.15 & 0.03 & 0.18 & 0.23 & 1.17 \\
\hline
\end{tabular}

${ }^{a}$ The percentage of endogenous hominin DNA was calculated as the fraction of sequences of at least 35 bp that mapped to the human reference over the total number of sequences

${ }^{\mathrm{b}}$ The values $L_{1 \%}$ and $L_{10}$ refer to length cutoffs that limit the fraction of spurious alignment to under $1 \%$ and $10 \%$, respectively. Column 35 bp refers to the standard 35-bp length threshold. Values have been computed using sequences with terminal C-to-T changes only and disregarding sequences with indels

specimens (an incisor and a femur fragment) share significantly more derived alleles with the high-coverage genome of a Neandertal than with that of a Denisovan individual [8]. While this result concurred with the fact that the Middle Pleistocene Sima de los Huesos remains were discovered in the western part of the territory inhabited by Neandertals during the Late Pleistocene (Europe and Central Asia), it deviated from the mitochondrial tree [7], which groups the Sima de los Huesos hominins into a clade with Denisovans, who are thought to have inhabited large parts of Asia [25, 26].

To test whether the inclusion of data from shorter nuclear sequences would affect inferences about the phylogenetic position of the Sima de los Huesos specimens, we compared the results of the lineage assignment test (see the "Methods" section) obtained by using a 35 -bp cutoff, as previously published, and the $L_{10 \%}$ cutoffs determined here for all four specimens for which at least 1000 sequences from putatively deaminated DNA fragments were available (Fig. 4). For the femur fragment and the incisor, the inclusion of additional data strengthens the confidence of the Neandertal lineage assignment, and the significance of the assignment was highest when between 2.5 and $15.6 \%$ of spurious alignments were allowed (Additional file 1: Figure S5). This suggests that a spurious alignment proportion of around $10 \%$ can be tolerated for this analysis. We caution that such a high proportion of spurious alignments is not necessarily tolerable by other types of analysis and that similar tests need to be carried out to determine appropriate cutoffs.

As previously, one of the other Sima de los Huesos samples (Femur XIII) did not yield sufficient data for a confident lineage assignment and no additional data could be gained by applying the $L_{10 \%}$ cutoff. However, the fourth specimen, a molar, shows significantly higher allele sharing with the Neandertal than the Denisovan genome with the $L_{10 \%}$ cutoff (Fig. 4 , Fisher's exact test $p$ value $=0.005$ corrected for multiple testing [27]). Moreover, the percentage of Neandertal-shared derived alleles of the molar (35\%) does not significantly differ from the percentages observed for the incisor and the femur fragment (43\% and 39\%, respectively; all pairwise Fisher's exact tests $p$ values $>0.29)$.

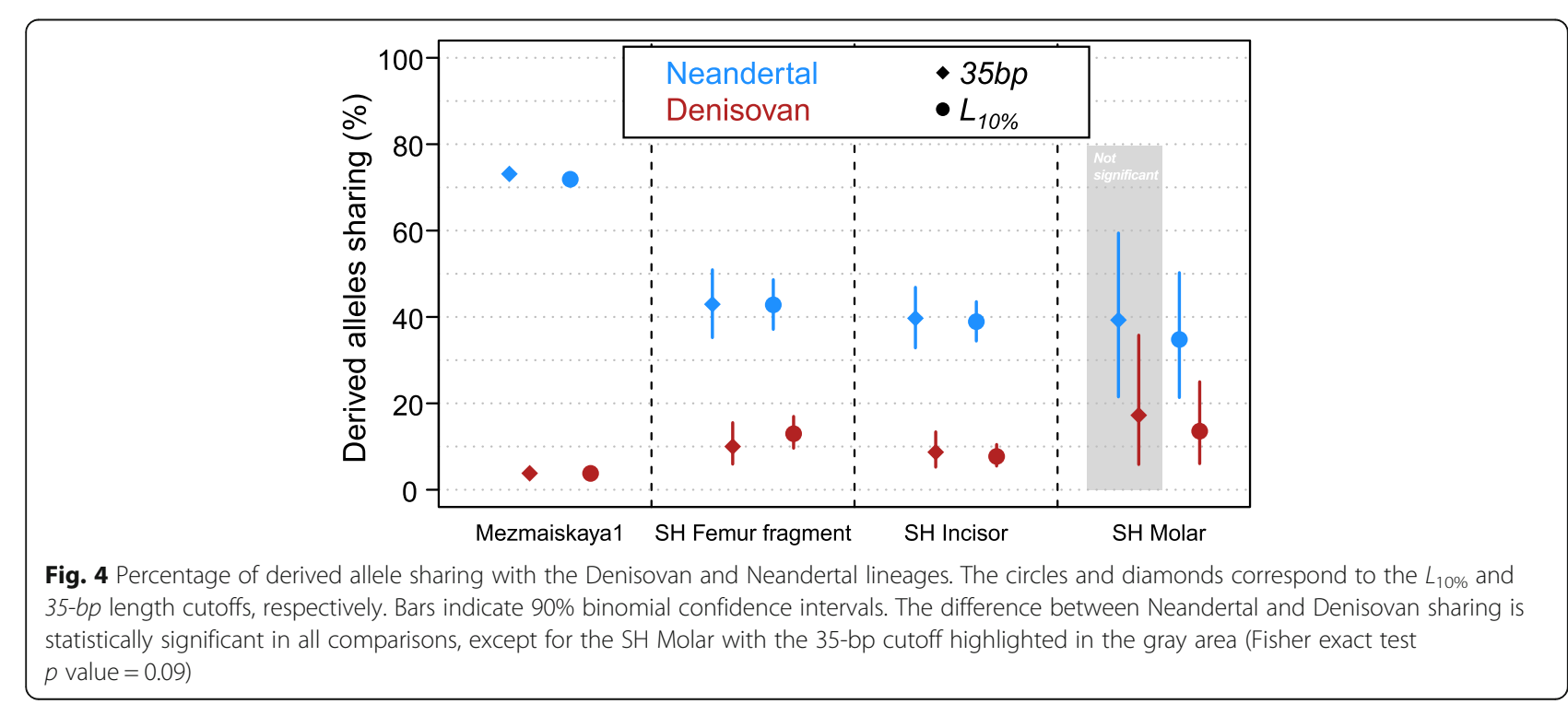




\section{Phylogenetic inferences and reference bias}

Since the fraction of mismatches in alignments is limited, spuriously aligning sequences are expected to exhibit a strong bias towards showing the human reference allele. This preference for the human reference allele should introduce a bias towards supporting the modern human lineage in the lineage assignment analysis of spurious alignments. In agreement with this expectation, we observe a strong bias towards the human reference allele in misaligning bacterial sequences, which are assigned to the modern human lineage $(\sim 33 \%$ of the human derived variants shared). A similar signal is also observed for the lineage assignment of Sima de los Huesos when considering size cutoffs that are expected to lead to an overwhelming majority of spurious alignments (Additional file 1: Figure S6). While our results with the $L_{10 \%}$ cutoffs do not show significant differences to previous, more conservative cutoffs for these samples, we caution that reference bias may affect analyses and needs to be considered before including a higher fraction of spurious alignments.

\section{Discussion}

Experimental procedures have made great strides forward in extracting short ancient DNA fragments $[3,5,6]$. However, the resulting short sequences constitute a challenge for computational processing since unrelated and related sequences cannot easily be distinguished. This has led to the paradoxical situation, in which short DNA fragments that are preserved in highly degraded samples can be made accessible to sequencing, only to be discarded in downstream computational analyses to avoid spurious alignments.

How can shorter sequences be made available for analysis without increasing the fraction of spurious alignments unduly? We have shown here that one answer lies in specific filters that enrich for genuine alignments. By filtering for sequences with evidence for deamination and without insertion/deletion differences to the reference genome, we were able to reduce the fraction of spurious alignments sufficiently to allow for the inclusion of sequences shorter than $35 \mathrm{bp}$ from three Sima de los Huesos samples in phylogenetic analysis. This analysis confirmed that two of the samples originate from early Neandertals and enabled us to place one additional sample, a molar, on the Neandertal lineage. The Neandertal allele sharing of this sample is similar to that of the other two. All three samples could thus originate from a single group of early Neandertal ancestors or relatives thereof.

The highly degraded remains from Sima de los Huesos yielded, arguably, the most challenging dataset in ancient DNA to date, containing a large fraction of ultra-short sequences and a large fraction of sequences from microbial contamination. In light of these difficulties, it is encouraging for future work on material with poor DNA preservation that useful genetic information could be recovered from ultra-short sequences of three samples from the site. However, we have to acknowledge that working with such sequences remains a challenge. Perhaps the best example of this is given by our analysis of a fourth Sima de los Huesos sample, FemurXIII, for which a minimum sequence length cutoff of $46 \mathrm{bp}$ must be applied to ensure that the fraction of spurious alignments is restricted to less than $1 \%$. This result shows that microbial contamination is so abundant in this sample that the commonly used cutoffs of $35 \mathrm{bp}$ length or shorter (Additional file 1: Table S5) is insufficient to reduce the effect of spurious alignments to conservative levels. As more data from highly degraded material become available, it will be crucial to ensure that spurious alignments are quantified to avoid false results.

On a broader level, our results show that the genetic analysis of poorly preserved ancient biological material is limited not only by our ability to extract and sequence the DNA it may contain, but also by our ability to distinguish sequences that are endogenous to the organism from the overwhelming majority of microbial contamination. Molecular methods have been developed in the past to decrease the fraction of microbial contamination. These methods used restriction enzymes that cut motifs occurring preferentially in contaminant DNA [16], enriched for endogenous DNA fragments via hybridization capture [28] or depleted contaminant DNA prior to DNA extraction $[29,30]$. Further research will be needed to establish how these methods can contribute to the study of highly degraded samples.

\section{Conclusions}

We conclude that while spurious alignments are an inevitable issue for the analysis of short ancient sequences, their influence can be accurately assessed and limited by appropriate filtering. Together with further refinement of molecular methods our approach paves the way towards the study of older or more degraded samples.

\section{Methods \\ Modifying the human reference genome}

The human reference genome (hg19/GRCh37) was used as a template to create a genome with additional single nucleotide changes. These changes were introduced in conserved regions where the reference human base is identical to the aligned bases of the chimpanzee pantro4 genome, the high-coverage genomes of the Altai Neandertal [20] and the Denisovan [6], 24 high-coverage modern human genomes $[6,20]$, and all 2504 modern human individuals of the 1000 Genomes Project data phase 3 [31]. Sites 5-bp up- and downstream of all indels detected in these datasets were excluded. Sites were also required to fall outside of simple repeats annotated using the Tandem Repeat 
Finder [32] and to overlap positions of unique mappability based on 35mers [20]. Bases were changed every $100 \mathrm{bp}$. If a change fell in a region that was excluded, the closest included position was determined and chosen as new location if it was at least $75 \mathrm{bp}$ from the closest adjacent changed site. Bases were replaced by other bases according to probabilities that keep the overall nucleotide composition identical to that of the hg19 genome. A total of 18,002,060 sites were modified.

\section{Sequence data and alignments to the modified reference} We used one lane of Illumina HiSeq 2500 sequencing data from the Mezmaiskaya1 Neandertal individual (library R5661; see Suppl. 2 in ref. [21]) and the published sequences from Sima de los Huesos samples [8] Femur fragment, Incisor, Molar, and FemurXIII. Both datasets were generated with the same extraction method [3] and the single stranded DNA library protocol [33].

As negative control-i.e., as a sample for which we do not expect to see any spurious alignments-we used modern human DNA that was sheared to short fragments of similar size to those in ancient samples. In details, DNA was extracted from the blood of a healthy human donor using the Gentra Puregene Blood Kit (Qiagen). One microgram of DNA was sheared for $2 \mathrm{~h}$ using the Covaris S2 ultrasonicator (shearing parameters: intensity 5 , cycler/burst 1000 , duty cycles $10 \%$ ) to obtain a fragment size distribution that mimics that of ancient DNA. A 200-ng aliquot of sheared DNA was then used as input for silica-based DNA extraction [3]. A single-stranded library [33] was prepared from $2.5 \mu \mathrm{l}$ of the resulting DNA extract (5\% of the extract). The library was amplified using Accuprime Pfx DNA polymerase (Thermo Fisher Scientific) [34] and a pair of indexing primers containing a sample-specific combination of 7-bp indices [35]. The indexed library was sequenced on 6 lanes of a HiSeq 2000 (Illumina) in $2 \times 76$ bp paired-end configuration with two index reads [35]. Sequences without perfect matches to the expected index combination were discarded. Subsequent processing was carried out identically to the Mezmaiskaya 1 and Sima de los Huesos data.

For our positive control-i.e. a sample with solely spurious alignments-we used 3860 bacteria genomes from the European Nucleotide Archive listed here http://www.ebi.ac.uk/genomes/bacteria.details.txt. The genomes were then fragmented from 20 to $40 \mathrm{bp}$ with approximatively the same number of sequences at each sequence length. This resulted in a total of $\sim 3.03$ billion unique sequences (Additional file 1: Table S1). Given that bacteria might not be the only organisms representing the environmental contamination, we also used two eukaryotic genomes. These are a fungus (Saccharomyces cerevisiae, "sacCer3" S288c strain assembly from GCA_000146055.2) and a protist (Albugo laibachii, NCBI:txid653948), which resulted in a total of $\sim 9.66$ and $\sim 32.04$ million sequences, respectively (Additional file 1: Tables S2 and S3). Ambiguous bases were replaced with one randomly chosen representative base.

All sequence data were mapped to the modified human reference genome using bwa [36] with options "-n 0.01 -o 2 -1 16500" matching those used for the ancient samples $[6,14]$. Sequences were merged when they appeared to originate from a PCR duplicate by means of bam-rmdup (https://bitbucket.org/ustenzel/ biohazard-tools). Paired-end sequences and sequences shorter than $20 \mathrm{bp}$ were disregarded.

\section{Length-dependent mappability tracks}

We used the software GEM [37] to generate maps of unique mappability of different lengths for the human reference genome (GCRh37/hg19) including decoy sequences [31]. The program was run for lengths of 20, $23,26,29,32$, and 35 bp allowing for up to one mismatch in alignments. To determine whether a sequence was mappable, we first chose the largest mappability track that was not longer than the sequence length. The sequence was deemed uniquely aligned if it contained a uniquely mappable motif in the reference within its alignment. All analyses involve this filtering.

\section{Features of spurious alignments}

Sequences that mapped to the modified reference genome and overlap mutated sites were used to determine characteristics of spurious and true alignments. Alignments were classified as true if they showed the human reference base and as spurious if they showed the mutated variant or any other allele than the human reference. For both spurious and true alignments, we calculated:

1) The proportion of mismatches, i.e., the number of observed mismatches relative to the modified reference genome divided by sequence length. For sequences that did not match the modified reference's allele, we subtracted one mismatch to compensate for the mismatch caused by the artificially mutated site. This correction was applied to true and spurious alignments, alike.

2) The number of insertion and deletions (indels), extracted from the CIGAR field in the bam/sam format files.

3) The patterns of nucleotide substitutions, determined by comparing the sequences to the unmodified hg19 reference.

To minimize the impact of cytosine deamination, we make use of the preserved strand orientation of sequences 
prepared with the single-stranded library protocol [33] and disregarded alignments in the forward orientation if either the mutated or original human reference state was $\mathrm{C}$, or alignments in reverse orientation if the mutated or original human reference state was G. Due to this filter, $37 \%$ of mutated sites (C-to-G or G-to-C) are disregarded.

\section{Quantifying the fraction of spurious alignments}

To calculate the proportion of spurious alignments, we make use of the number of alignments classified as truly related $\left(N_{\mathrm{T}}\right)$ and the number of alignments classified as spurious $\left(N_{\neg \mathrm{T}}\right)$ as described in the previous section. A small fraction of spurious alignments is expected to show the human reference base by chance. To correct for this, we assume that all spurious alignments contain the maximum number of mismatches. The maximum proportion of mismatches for a sequence is $M=m / l$ where $m$ denotes the maximum number of mismatches allowed in a sequence of length $l$. Only a third of the exchanges at any given position will match the original reference base, so that the probability for a spuriously aligning sequence to show the reference base is at most $M \times 1 / 3$. We then conservatively correct the $N_{\mathrm{T}}$ and $N_{\neg \mathrm{T}}$ counts to compensate for spurious misclassified alignments by calculating:

$$
\begin{aligned}
& N_{\mathrm{T}}^{\prime}=N_{\mathrm{T}}-N_{\neg \mathrm{T}} \frac{M}{3-M} \\
& N_{\neg \mathrm{T}}^{\prime}=\frac{N_{\neg \mathrm{T}}}{1-\frac{M}{3}}
\end{aligned}
$$

With these corrected counts, we calculate the spurious alignment proportion as:

$$
\frac{N_{\neg \mathrm{T}}^{\prime}}{N_{\neg \mathrm{T}}^{\prime}+N_{\mathrm{T}}^{\prime}}
$$

\section{Lineage assignment}

Informative sites were determined by sampling one random allele from each of the genotypes of a modern human (Mbuti, HGDP0456 in [20]), the Altai Neandertal and the Denisovan genomes after applying the minimum set of filters described in [20]. For the Altai Neandertal and Denisovan genomes [21], we used the most recent genotype calls by means of snpAD [38] instead of those of the first publications $[6,20]$. To call the ancestral state at each site, we used whole genome alignments of five primates (pantro4, bonobo, gorgor3, ponabe2, and rhemac2) to the human reference, and required that at least four of them agree. Derived sites were assigned to the following four lineages: Modern Human, Neandertal, Denisovan, and Neandertal-Denisovan.
For each dataset, we iterated overall sequences, aligned to the unmodified hg19 reference, and calculated the percentage of derived alleles of each class that are shared. All $\mathrm{T}$ within the last three terminal positions of sequences were disregarded to minimize the impact of C-to- $\mathrm{T}$ changes due to deamination.

\section{Additional file}

Additional file 1: Table S1. Summary of bacterial sequences. Table S2. Summary of S. cerevisiae sequences. Table S3. Summary of A. laibachii sequences. Table S4. Percentage of sequences with a given number of mismatches for each type of alignments. Table S5. Sequence length cutoffs in hominin ancient DNA studies [39-65]. Table S6. Modern human contamination. Figure S1. Characteristics of spurious and true alignments. Figure S2. Length distribution of mapped sequences. Figure S3. Proportion of spurious alignments by sequence length for $\mathrm{SH}$ samples. Figure S4. Spurious alignments in SH samples and the effect of different filters. Figure S5. Significance of Neandertal-lineage assignment for different minimum length cutoffs. Figure S6. Lineage assignment as a function of sequence length. (PDF $767 \mathrm{~kb}$ )

\section{Acknowledgements}

We are thankful to Michael Dannemann, Janet Kelso, Fabrizio Mafessoni, Svante Pääbo, Frédéric Romagné, Udo Stenzel, and the Neandertal and Bioinformatics groups of the Evolutionary Genetics department for the helpful discussions and suggestions during the development of the project. We are also grateful to Marie Gansauge and Birgit Nickel for the extraction and library preparation of the modern human sample. We are also grateful to three anonymous reviewers for their helpful comments.

\section{Funding}

This study was supported by the Max Planck Society and funded by the Max Planck Foundation grant "No. 31-12LMP Pääbo" and by the European Research Council grant No. 694707 to Svante Pääbo.

\section{Availability of data and materials}

All data generated or analyzed during this study are included in this published article and its supplementary information files. The description of the pipeline, the software and scripts, and the modified human reference genome are available in https://figshare.com/projects/SpAl/39137 and can also be found under http://bioinf.eva.mpg.de/SpAl/.

\section{Authors' contributions}

MM and KP designed the study. All authors analyzed the data, interpreted the results, and wrote the manuscript. All authors read and approved the final manuscript.

\section{Ethics approval and consent to participate}

The sequencing of human DNA as a control for ancient DNA was approved by the Ethik-Komission of the Medical Faculty of the University of Leipzig (sign: 364-14-17112014)

Consent for publication

Not applicable

\section{Competing interests}

The authors declare that they have no competing interests

\section{Publisher's Note}

Springer Nature remains neutral with regard to jurisdictional claims in published maps and institutional affiliations. 
Received: 15 August 2018 Accepted: 27 September 2018 Published online: 25 October 2018

\section{References}

1. Dabney J, Meyer M, Pääbo S. Ancient DNA damage. Cold Spring Harb Perspect Biol. 2013:5:a012567.

2. Allentoft ME, Collins M, Harker D, Haile J, Oskam CL, Hale ML, et al. The halflife of DNA in bone: measuring decay kinetics in 158 dated fossils. Proc Biol Sci. 2012;279:4724-33.

3. Dabney J, Knapp M, Glocke I, Gansauge M-T, Weihmann A, Nickel B, et al. Complete mitochondrial genome sequence of a Middle Pleistocene cave bear reconstructed from ultrashort DNA fragments. Proc Natl Acad Sci U A S. 2013;110:15758-63.

4. Allentoft ME, Sikora M, Sjögren K-G, Rasmussen S, Rasmussen M, Stenderup J, et al. Population genomics of Bronze Age Eurasia. Nature. 2015:522:167-72.

5. Glocke I, Meyer M. Extending the spectrum of DNA sequences retrieved from ancient bones and teeth. Genome Res. 2017;27:1230-7.

6. Meyer M, Kircher M, Gansauge M-T, Li H, Racimo F, Mallick S, et al. A highcoverage genome sequence from an archaic Denisovan individual. Science. 2012;338:222-6.

7. Meyer M, Fu Q, Aximu-Petri A, Glocke I, Nickel B, Arsuaga J-L, et al. A mitochondrial genome sequence of a hominin from Sima de los Huesos. Nature. 2014;505:403-6.

8. Meyer M, Arsuaga J-L, de Filippo C, Nagel S, Aximu-Petri A, Nickel B, et al. Nuclear DNA sequences from the Middle Pleistocene Sima de los Huesos hominins. Nature. 2016:531:504

9. Noonan JP, Hofreiter M, Smith D, Priest JR, Rohland N, Rabeder G, et al. Genomic sequencing of Pleistocene cave bears. Science. 2005;309:597-9.

10. Green RE, Krause J, Ptak SE, Briggs AW, Ronan MT, Simons JF, et al. Analysis of one million base pairs of Neanderthal DNA. Nature. 2006:444:330-6.

11. Poinar HN, Schwarz C, Qi J, Shapiro B, Macphee RDE, Buigues B, et al. Metagenomics to paleogenomics: large-scale sequencing of mammoth DNA. Science. 2006:311:392-4.

12. Der Sarkissian C, Allentoft ME, Ávila-Arcos MC, Barnett R, Campos PF, Cappellini E, et al. Ancient genomics. Philos Trans R Soc Lond Ser B Biol Sci. 2015;370:20130387

13. Prüfer K, Stenzel U, Hofreiter M, Pääbo S, Kelso J, Green RE. Computational challenges in the analysis of ancient DNA. Genome Biol. 2010;11:R47.

14. Kircher M. Analysis of high-throughput ancient DNA sequencing data. In: Shapiro B, Hofreiter M, editors. Ancient DNA: methods and protocols. Totowa: Humana Press; 2012. p. 197-228. https://doi.org/10.1007/978-161779-516-9_23.

15. Smith TF, Waterman MS, Burks C. The statistical distribution of nucleic acid similarities. Nucleic Acids Res. 1985;13:645-56.

16. Green RE, Krause J, Briggs AW, Maricic T, Stenzel U, Kircher M, et al. A draft sequence of the Neandertal genome. Science. 2010;328:710-22.

17. Schubert M, Ginolhac A, Lindgreen S, Thompson JF, Al-Rasheid KAS,

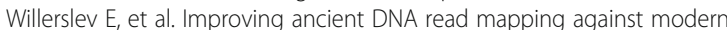
reference genomes. BMC Genomics. 2012;13:178.

18. Bennett EA, Massilani D, Lizzo G, Daligault J, Geigl E-M, Grange T. Library construction for ancient genomics: single strand or double strand? BioTechniques. 2014;56:289-300.

19. Renaud G, Hanghøj K, Willerslev E, Orlando L. gargammel: a sequence simulator for ancient DNA. Bioinformatics. 2017:33:577-9.

20. Prüfer K, Racimo F, Patterson N, Jay F, Sankararaman S, Sawyer S, et al. The complete genome sequence of a Neanderthal from the Altai Mountains. Nature. 2014;505:43-9.

21. Prüfer K, de Filippo C, Grote S, Mafessoni F, Korlević P, Hajdinjak M, et al. A high-coverage Neandertal genome from Vindija Cave in Croatia. Science. 2017;358:655-8.

22. Briggs AW, Stenzel U, Johnson PLF, Green RE, Kelso J, Prüfer K, et al. Patterns of damage in genomic DNA sequences from a Neandertal. Proc Natl Acad Sci U S A. 2007:104:14616-21.

23. Campbell CD, Eichler EE. Properties and rates of germline mutations in humans. Trends Genet. 2013;29:575-84.

24. Green RE, Briggs AW, Krause J, Prüfer K, Burbano HA, Siebauer M, et al. The Neandertal genome and ancient DNA authenticity. EMBO J. 2009:28:2494-502.

25. Reich D, Green RE, Kircher M, Krause J, Patterson N, Durand EY, et al. Genetic history of an archaic hominin group from Denisova cave in Siberia. Nature. 2010;468:1053-60.
26. Reich D, Patterson N, Kircher M, Delfin F, Nandineni MR, Pugach I, et al. Denisova admixture and the first modern human dispersals into Southeast Asia and Oceania. Am J Hum Genet. 2011;89:516-28.

27. Benjamini $Y$, Hochberg $Y$. Controlling the false discovery rate: a practical and powerful approach to multiple testing. J R Stat Soc Ser B Stat Methodol. 1995:57:289-300.

28. Fu Q, Meyer M, Gao X, Stenzel U, Burbano HA, Kelso J, et al. DNA analysis of an early modern human from Tianyuan Cave, China. Proc Natl Acad Sci U S A. 2013:110:2223-7.

29. Damgaard PB, Margaryan A, Schroeder H, Orlando L, Willerslev E, Allentoft ME. Improving access to endogenous DNA in ancient bones and teeth. Sci Rep. 2015;5:11184.

30. Korlević P, Gerber T, Gansauge M-T, Hajdinjak M, Nagel S, Aximu-Petri A, et al. Reducing microbial and human contamination in DNA extractions from ancient bones and teeth. BioTechniques. 2015:59:87-93.

31. 1000 Genomes Project Consortium, Auton A, Brooks LD, Durbin RM, Garrison EP, Kang HM, et al. A global reference for human genetic variation. Nature. 2015;526:68-74.

32. Benson G. Tandem repeats finder: a program to analyze DNA sequences. Nucleic Acids Res. 1999;27:573-80.

33. Gansauge M-T, Meyer M. Single-stranded DNA library preparation for the sequencing of ancient or damaged DNA. Nat Protoc. 2013;8:737-48.

34. Dabney J, Meyer M. Length and GC-biases during sequencing library amplification: a comparison of various polymerase-buffer systems with ancient and modern DNA sequencing libraries. BioTechniques. 2012;52:87-94.

35. Kircher M, Sawyer S, Meyer M. Double indexing overcomes inaccuracies in multiplex sequencing on the Illumina platform. Nucleic Acids Res. 2012;40:e3.

36. Li H, Durbin R. Fast and accurate short read alignment with BurrowsWheeler transform. Bioinformatics. 2009:25:1754-60.

37. Marco-Sola S, Sammeth M, Guigó R, Ribeca P. The GEM mapper: fast, accurate and versatile alignment by filtration. Nat Methods. 2012;9:1185-8.

38. Prüfer K. snpAD: an ancient DNA genotype caller. Bioinformatics. 2018 https://doi.org/10.1093/bioinformatics/bty507.

39. Malaspinas A-S, Lao O, Schroeder H, Rasmussen M, Raghavan M, Moltke I, et al. Two ancient human genomes reveal Polynesian ancestry among the indigenous Botocudos of Brazil. Curr Biol. 2014;24:1035-7.

40. Raghavan M, Skoglund P, Graf KE, Metspalu M, Albrechtsen A, Moltke I, et al. Upper Palaeolithic Siberian genome reveals dual ancestry of Native Americans. Nature. 2014:505:87-91.

41. Martiniano R, Caffell A, Holst M, Hunter-Mann K, Montgomery J, Müldner G, et al. Genomic signals of migration and continuity in Britain before the Anglo-Saxons. Nat Commun. 2016;7:10326.

42. Raghavan M, Steinrücken M, Harris K, Schiffels S, Rasmussen S, DeGiorgio M, et al. Genomic evidence for the Pleistocene and recent population history of Native Americans. Science. 2015;349:aab3884

43. Rasmussen M, Guo X, Wang Y, Lohmueller KE, Rasmussen S, Albrechtsen A, et al. An aboriginal Australian genome reveals separate human dispersals into Asia Science 2011:334:94-8.

44. Gamba C, Jones ER, Teasdale MD, McLaughlin RL, Gonzalez-Fortes G, Mattiangeli $\mathrm{V}$, et al. Genome flux and stasis in a five millennium transect of European prehistory. Nat Commun. 2014;5:5257.

45. Olalde I, Allentoft ME, Sánchez-Quinto F, Santpere G, Chiang CWK, DeGiorgio $M$, et al. Derived immune and ancestral pigmentation alleles in a 7,000-year-old Mesolithic European. Nature. 2014:507:225-8.

46. Gallego Llorente M, Jones ER, Eriksson A, Siska V, Arthur KW, Arthur JW, et al. Ancient Ethiopian genome reveals extensive Eurasian admixture throughout the African continent. Science. 2015;350:820-2.

47. Jones ER, Gonzalez-Fortes G, Connell S, Siska V, Eriksson A, Martiniano R, et al. Upper Palaeolithic genomes reveal deep roots of modern Eurasians. Nat Commun. 2015;6:8912

48. Olalde I, Schroeder H, Sandoval-Velasco M, Vinner L, Lobón I, Ramirez O, et al. A common genetic origin for early farmers from Mediterranean Cardial and Central European LBK cultures iterranean Cardial and Centra European LBK cultures. Mol Biol Evol. 2015;32:3132-42.

49. Rasmussen M, Sikora M, Albrechtsen A, Korneliussen TS, Moreno-Mayar JV, Poznik GD, et al. The ancestry and affiliations of Kennewick Man. Nature. 2015;523:455-8.

50. Hofmanová Z, Kreutzer S, Hellenthal G, Sell C, Diekmann Y, Díez-Del-Molino $D$, et al. Early farmers from across Europe directly descended from Neolithic Aegeans. Proc Natl Acad Sci U S A. 2016;113:6886-91. 
51. Cassidy LM, Martiniano R, Murphy EM, Teasdale MD, Mallory J, Hartwell B, et al. Neolithic and Bronze Age migration to Ireland and establishment of the insular Atlantic genome. Proc Natl Acad Sci U S A. 2016;113:368-73.

52. Fu Q, Li H, Moorjani P, Jay F, Slepchenko SM, Bondarev AA, et al. Genome sequence of a 45,000-year-old modern human from western Siberia. Nature. 2014;514:445-9.

53. Lazaridis I, Patterson N, Mittnik A, Renaud G, Mallick S, Kirsanow K, et al. Ancient human genomes suggest three ancestral populations for presentday Europeans. Nature. 2014;513:409-13.

54. Skoglund P, Malmström H, Omrak A, Raghavan M, Valdiosera C, Günther T, et al. Genomic diversity and admixture differs for Stone-Age Scandinavian foragers and farmers. Science. 2014;344:747-50.

55. Fu Q, Hajdinjak M, Moldovan OT, Constantin S, Mallick S, Skoglund P, et al. An early modern human from Romania with a recent Neanderthal ancestor. Nature. 2015;524:216-9.

56. Günther T, Valdiosera C, Malmström H, Ureña I, Rodriguez-Varela R, Sverrisdóttir ÓO, et al. Ancient genomes link early farmers from Atapuerca in Spain to modern-day Basques. Proc Natl Acad Sci U A. 2015;112:11917-22.

57. Schlebusch CM, Malmström H, Günther T, Sjödin P, Coutinho A, Edlund H, et al. Southern African ancient genomes estimate modern human divergence to 350,000 to 260,000 years ago. Science. 2017;358:652-5.

58. Skoglund $\mathrm{P}$, Thompson JC, Prendergast ME, Mittnik A, Sirak K, Hajdinjak M, et al. Reconstructing Prehistoric African Population Structure. Cell. 2017;171:59-71 e21.

59. Hajdinjak M, Fu Q, Hübner A, Petr M, Mafessoni F, Grote S, et al. Reconstructing the genetic history of late Neanderthals. Nature. 2018;555:652-6.

60. Rasmussen M, Li Y, Lindgreen S, Pedersen JS, Albrechtsen A, Moltke I, et al. Ancient human genome sequence of an extinct Palaeo-Eskimo. Nature. 2010;463:757-62.

61. Keller A, Graefen A, Ball M, Matzas M, Boisguerin V, Maixner F, et al. New insights into the Tyrolean Iceman's origin and phenotype as inferred by whole-genome sequencing. Nat Commun. 2012;3:698.

62. Rasmussen M, Anzick SL, Waters MR, Skoglund P, DeGiorgio M, Stafford TW $\mathrm{Jr}$, et al. The genome of a Late Pleistocene human from a Clovis burial site in western Montana. Nature. 2014;506:225-9.

63. Seguin-Orlando A, Korneliussen TS, Sikora M, Malaspinas A-S, Manica A, Moltke I, et al. Paleogenomics. Genomic structure in Europeans dating back at least 36,200 years. Science. 2014;346:1113-8.

64. Schiffels S, Haak W, Paajanen P, Llamas B, Popescu E, Loe L, et al. Iron Age and Anglo-Saxon genomes from East England reveal British migration history. Nat Commun. 2016;7:10408

65. Sikora M, Seguin-Orlando A, Sousa VC, Albrechtsen A, Korneliussen T, Ko A, et al. Ancient genomes show social and reproductive behavior of early upper Paleolithic foragers. Science. 2017;358:659-62.

Ready to submit your research? Choose BMC and benefit from:

- fast, convenient online submission

- thorough peer review by experienced researchers in your field

- rapid publication on acceptance

- support for research data, including large and complex data types

- gold Open Access which fosters wider collaboration and increased citations

- maximum visibility for your research: over $100 \mathrm{M}$ website views per year

At $\mathrm{BMC}$, research is always in progress.

Learn more biomedcentral.com/submissions 